\title{
Acute kidney injury in neonatal intensive care: Medicines involved
}

\author{
A.I. Safina*, M.A. Daminova and G.A. Abdullina \\ Kazan State Medical Academy, Department of Pediatrics and Neonatology, Kazan, Russia
}

*Corresponding author. E-mail: safina_asia@mail.ru

BACKGROUND: The incidence of acute kidney injury (AKI) in neonates in the intensive care units and neonatal intensive care (NICU) according Plotz et al. ranges from $8 \%$ to $22 \%$ [3]. According to Andreoli, neonatal death due to AKI in NICU amounts up to $10-61 \%$ [1]. It should be in the reasons of AKI emphasize

The role of certain drugs, which are widely used in modern neonatology: nonsteroidal antiinflammatory drugs (NSAIDs), antibiotics (aminoglycosides, glycopeptides, carbapenems, 3rd generation cephalosporins), furosemide, enalapril, in contributing to AKI should be emphasized [2].

OBJECTIVE: To identify risk factors for acute kidney injury in neonates in intensive care units and intensive care.

METHODS: We performed a prospective observational case-control study of full-term newborns who were treated in the intensive care unit and neonatal intensive care of the "Children’s city hospital №1" Kazan and NICU №3 “Children’s Republican Clinical Hospital” in 2011-2014 years.

The study included 86 term infants in critical condition, who were hospitalized to the NICU on the first days of life, - the main group. The main criterion of AKI in neonates according to neonatal AKIN classification (2011) is a serum creatinine concentration $\geq 1.5 \mathrm{mg} / \mathrm{dL}$. We subdivided the main group into two subgroups:

subgroup I, AKI+ consisted of 12 term infants in critical condition with the serum creatinine level $\geq 1,5 \mathrm{mg} / \mathrm{dL}$ at the age of not younger than 48 hours after birth, which was $14 \%$ of all full-term newborns who were at the NICU;

subgroup II, AKI- consisted of 74 term infants in critical condition with the serum creatinine level $<1.5 \mathrm{mg} / \mathrm{dL}$ at the age of not younger than 48 hours after birth.

The control group was formed by random sampling, it consisted of 26 somatically healthy term infants. We used conventional methods for evaluating renal function, and enzyme immunoassay (ELISA) for the urine biomarker of AKI, IL-18.

Statistical analysis was performed using SPSS, Statistics 20, and the IBM and Microsoft Office Excel 2007. The study results were subjected to statistical analysis using parametric and non-parametric methods of analysis. We present the findings as arithmetic means (M) with, standard deviation $(\sigma)$ and standard error of the mean (m) according to standard formulas.

RESULTS: All children were admitted to primary and emergency care with subsequent transfer to the NICU at 1-2 days of life and further treatment in the department of pathology of newborns (DPN). The duration of hospitalization of infants at the NICU for the main group averaged 5,9 $\pm 0,44$ days; at the DPN (subsequent stage of nursing) - 11,42 $\pm 0,51$ days; for the subgroup I, AKI+ newborns these 
were 7,83 $\pm 1,23$ days and 13,75 $\pm 3,34$ days, respectively; for the subgroup II, AKI- newborns these were 5,58 $\pm 0,47$ (3-30) and 11,04 $\pm 0,3(0-47)$ days, respectively. Neonates received daily average of $16,5 \pm 0,3$ various medicines while at NICU and 9,1 $\pm 0,7$ while at DPN. Overall, over the entire period of hospitalization neonates of the main group received on average $25,6 \pm 1,8$ medicines. Of these, $2,9 \pm 0,4$ drugs were antibiotics, possessing nephrotoxic properties (aminoglycosides, cephalosporins, carbapenems, fluoroquinolones). Children of the main group in $100 \%(n=86)$ of cases were treated with 3rd generation cephalosporins (ceftriaxone, cephaperazone/sulbactam (sulperazon)), in 55\% od cases $(n=47)$ - with aminoglycosides (amikacin, gentamicin), in 1\% $(n=1)$ - with vancomycin, in 7\% $(n=6)$ - with carbapenems. Diuretics were prescribed to $57 \%(n=49)$ of infants. Often, patients were treated with a combination of nephrotoxic medications. Aminoglycoside were prescribed statistically more often to neonates of the subgroup I, than of the subgroup II $(p<0.01)$. Diuretic drugs were used more frequently and for longer periods of time in neonates of the subgroup I $(\mathrm{AKI}+)$, than in newborns of the subgroup II (AKI-), namely, in $83 \%(n=10)$ for $4,6 \pm 1.34$ days versus $53 \%$ of cases $(n=39)$ for $2.84 \pm 0.49$ days, respectively $(p<0.05)$. IL-18 urine level in neonates of subgroup I $(\mathrm{AKI}+)$ was 2 times higher than that in neonates of the subgroup II (AKI-), and 13 times higher than in neonates of the control group.

The fact that the IL-18 urine level increased with progression of kidney damage, caused by nephrotoxic therapy, suggests that a significant role in the development and progression of AKI in neonates at NICU belongs to drug therapy.

CONCLUSIONS: Full-term newborns in intensive care units are at high risk of AKI when they are treated with aminoglycosides in combination with diuretics for longer than 4.5 days.

Keywords: Risk factor, acute kidney injury, neonates, intensive care

Conflict of interest statement: The study did not have sponsorship. Authors are fully responsible for the provision of the final version of the manuscript for publication. All authors participated in the conception and design of the study and writing of the manuscript. The final version of the manuscript was approved by all authors. The authors did not receive a fee for the study.

\section{References}

[1] Andreoli SP. Acute renal failure in the newborn. Semin Perinatol. 2004;28 (2): 112-3. doi:10.1053/j.semperi.2003.11.003

[2] Morgan CJ, Zappitelli M, Robertson CM et al. Risk factors for and outcomes of acute kidney injury in neonates undergoing complex cardiac surgery. J Pediatr. 2013;162(1):120-7.e1. doi: 10.1016/j.jpeds.2012.06.054. Epub 2012 Aug 9.

[3] Plotz FB, Bouma AB, van Wijk JA, Kneyber MC, Bokenkamp A. Pediatric acute kidney injury in the ICU: An independent evaluation of pRIFLE criteria. Intensive Care Med. 2008;34(9):1713-7. doi: 10.1007/s00134-008-1176-7. Epub 2008 Jun 3. 